\title{
Transition of Planar Couette Flow at Infinite Reynolds Numbers
}

\author{
Tomoaki Itano* \\ Department of Pure and Applied Physics, Faculty of Engineering Science, Kansai University, Osaka 564-8680, Japan \\ Takeshi Akinaga and Sotos C. Generalis ${ }^{\dagger}$ \\ School of Engineering and Applied Sciences, Mathematics, Aston University, Birmingham B4 7ET, United Kingdom \\ Masako Sugihara-Seki \\ Department of Pure and Applied Physics, Faculty of Engineering Science, Kansai University, Osaka 564-8680, Japan
}

(Received 12 June 2013; published 30 October 2013)

\begin{abstract}
An outline of the state space of planar Couette flow at high Reynolds numbers $\left(\operatorname{Re}<10^{5}\right)$ is investigated via a variety of efficient numerical techniques. It is verified from nonlinear analysis that the lower branch of the hairpin vortex state (HVS) asymptotically approaches the primary (laminar) state with increasing Re. It is also predicted that the lower branch of the HVS at high Re belongs to the stability boundary that initiates a transition to turbulence, and that one of the unstable manifolds of the lower branch of HVS lies on the boundary. These facts suggest HVS may provide a criterion to estimate a minimum perturbation arising transition to turbulent states at the infinite Re limit.
\end{abstract}

DOI: 10.1103/PhysRevLett.111.184502

PACS numbers: 47.27.De, 47.20.Ky, 47.27.nd

Introduction.-Astronomers have found a tremendous number of stars far away from Earth in attempting to map our Universe, which is expanding due to the presence of the dark energy. Similarly, fluid physicists have recently elucidated that there exist a number of exact and invariant solutions in the state space of the turbulent shear flows governed by incompressible Navier-Stokes equations that will map turbulence [1-4]. The first discovery of an invariant finite-amplitude solution distinct from the laminar state, which is referred to hereafter as "Nagata-BusseWaleffe (NBW)" formed from the names of the discoverers [5-7], was achieved in a simple shear flow between parallel plates moving in opposite directions, planar Couette flow (PCF), with periodic boundary conditions in streamwise and spanwise directions and the following governing equation:

$$
\partial_{t} \boldsymbol{u}+\boldsymbol{u} \cdot \boldsymbol{\nabla} \boldsymbol{u}=-\boldsymbol{\nabla} p+\frac{1}{\operatorname{Re}} \nabla^{2} \boldsymbol{u},\left.\quad \boldsymbol{u}\right|_{y= \pm 1}= \pm \boldsymbol{e}_{x} .
$$

PCF is a prototype of canonical shear flow with a subcritical transition to turbulence, where a Newtonian fluid between two counter-sliding parallel plates at $y= \pm 1$ could yield at any Reynolds number (Re) the laminar state that consists only of the streamwise component, $\boldsymbol{u}=y \boldsymbol{e}_{x}$, and that is linearly stable for all finite Re (Ref. [8]). NBW emerges at a rather lower $\operatorname{Re}(\operatorname{Re}=127.7)$ than the one that is required for sustaining the turbulent regime in PCF, $\operatorname{Re} \gtrsim 325$, where coherent structures are localized (see Refs. $[9,10])$.

Wang et al. in Ref. [11] recently calculated that the NBW, amongst a number of solutions, is not just an exact solution emerging at the low Re, but also a significant "key" required to envisage the ideal turbulent regime at infinite Re from the physical point of view, by pointing out the following facts. First, NBW remains on the basin boundary (BB) even at high Re. BB is the hypersurface of codimension 1 in the state space associated with PCF, which separates the whole space into the basins of the laminar and the turbulent attractions. Second, NBW contains the only unstable manifold at high $\mathrm{Re}$, which escapes from the BB towards either the laminar or the turbulent attractions, so that NBW is an attractor of the dynamical system restricted on the BB. These facts make us envisage that NBW could yield an ultimate structure prevalent in the turbulent regime at the infinite Re, even if it has an instability along the unstable manifold towards turbulent attraction. It seems to be relevant to large-scale structures identified numerically in turbulent shear flows at high Re [12,13].

By contrast, within a classical picture, hairpin-shaped vortices, with an $\Omega$-shaped head and a pair of counterrotating streamwise legs, have, so far, been recognized as one of prevalent vortex structures in turbulent shear flows [14-17]. Here, we have to keep in mind that the NBW does not satisfy the reflection symmetry with respect to the spanwise direction, which is common in vortices observed in a transition triggered initially with a perturbation on the laminar flow. The recent progress in numerical simulations is enabling us to educe vortex structures in turbulent boundary layers at high Re over a huge domain [18], in which neither NBW nor hairpin-shaped vortices are detected further downstream of the turbulent boundary layer. However, in the turbulent layer, which develops with increasing Re (based on the boundary thickness $\operatorname{Re}_{\theta}$ [18]), it is the hairpinshaped vortices that emerge primarily at the beginning of the transition with a relatively high Re. 
Apparently, among these arguments, there seems to be some fundamental contradiction on the envisaged ultimate structure in shear flow in the infinite Re; there arises a question "why the envisaged ultimate structure, NBW, does not prevail at the transition with high Re?" In the present Letter, we will give an answer to this question, with the aid of a transition scenario deduced from the asymptotic behavior of another fundamental solution among a number of exact steady solutions of PCF, the hairpin vortex state (HVS) [1,2].

Asymptotic behavior of HVS.-In the bifurcation diagram, HVS consists of the upper and lower branches arising from a saddle-node bifurcation at the turning point $\operatorname{Re}=139.2$. It should again be emphasised that the reflection symmetry to the spanwise direction is satisfied by HVS but not by NBW. NBW emerges from a slightly lower Re than HVS does, in spite of the fact that NBW bifurcates due to the breaking of the symmetry from HVS; in other words, NBW is a derivative (rather than the counterpart) of the HVS.

In particular, the upper branch of HVS yields hairpinshaped vortex structures aligned by stream- and spanwise staggered formations reminiscent of those seen in experiments [14], after which the solution was named. However, a radical change of the topology of its velocity field with increasing $\operatorname{Re}(\operatorname{Re}>400)$ gives rise to a complicated deformation of the vortex structure, which leads to enhancement of the fluid mixing between the moving plates, followed by a steep rise of the wall shear rate. While the upper branch of HVS has, thus, attracted much attention recently $([19,20])$, obviously owing to its characteristics such as spatial shape and staggered formation, the analysis of the lower branch of HVS has been neglected in the recent focus of research.

According to Wang et al. [11], the lower branch of NBW has an asymptotic behavior at high Re limit, which consists both of a nonvanishing streak component and of vanishing streamwise vortices. Here, the "streak component" is referred to as the streamwise-independent perturbation $u_{0}(y, z)$ from the mean component $\bar{u}(y)$ of PCF, which is maintained by the streamwise vortices of NBW developing into a kind of critical layer at high Re. This fact means that the distance of NBW from the laminar state in the state space, $\left\|\boldsymbol{u}_{N}-\boldsymbol{u}_{L}\right\|$, is kept to be finite (nonvanishing) at the infinite Reynolds limit, where $\boldsymbol{u}_{N}$ is the lower branch of NBW and $\boldsymbol{u}_{L}$ is the laminar state. Moreover, because the NBW is a derivative of HVS, which bifurcates from the laminar state via the secondary branch in the bifurcation diagram ([19]), NBW cannot directly connect to the laminar state even at the infinite Re limit in a natural sense. Therefore, NBW can "not bifurcate from infinity"[11], contrary to the title of Ref. [5].

In contrast with NBW, the lower branch of HVS may diminish with increasing Re. First, in the present Letter, by means of the continuation analysis (homotopy) of the
Newton-Raphson method, we traced the lower branch of HVS up to as high Re as needed. Fourier and modified Chebyshev expansions are employed, where truncation is set to a large enough level to confirm that the spectrum of energies descends by a few orders of magnitude. Several representative components of the lower branch of HVS are indicated in Fig. 1. Referring to the first figure of Ref. [11], the amplitude of the streak component, $\left|u_{0}(y, z)-\bar{u}(y)\right|$, in Fig. 1 is plotted explicitly for both states, NBW and HVS. The lower branch of HVS algebraically looses all the harmonic amplitudes including even its streak component with increasing $\mathrm{Re}$, so that it asymptotically approaches the laminar state, which is distinct from the behavior of NBW. As it were, HVS could bifurcate from the laminar state at the infinite Re, while NBW could not. However, it would contradict the fact that the steady mode of the Orr-Sommerfeld equation is not neutral in the limit of infinite Re.

HVS on BB.-It is an open question whether the lower branch of the more primitive solution than NBW, HVS, also lies on the $\mathrm{BB}$ at high Re. In the present Letter, employing the edge tracking [21], which was established originally as a tool in order to find out an unstable steady solution in a bistable system [22] such as PCF, we specify the relation between the lower branch of HVS and the BB of PCF. Thus, we followed the time-development of the flow starting from the lower branch of HVS, $\boldsymbol{u}_{H}$, with a small perturbation, restricting the flow to satisfy the reflection symmetry. In Fig. 2, the trajectories of the time development of flow are depicted by a component of the velocity field, which is zero for the laminar regime and of order 1

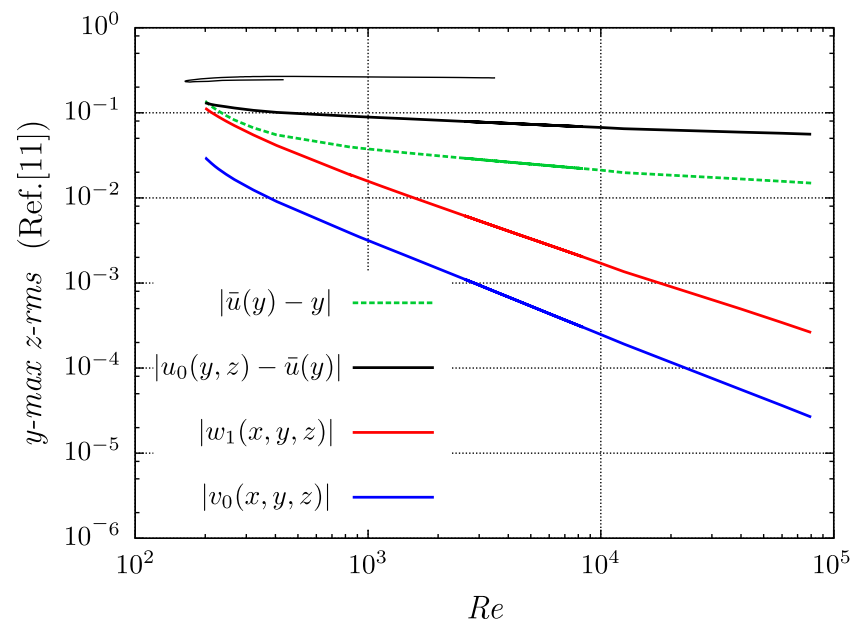

FIG. 1 (color online). Amplitudes of representative Fourier modes for the HVS in PCF are plotted against Re for stream- and spanwise extents of the periodic box $\left(L_{x}, L_{z}\right)=(2 \pi, \pi)$. All the amplitudes of the HVS algebraically decrease with increasing Re. In particular, gradual attenuation in the streak component $\left|u_{0}(y, z)-\bar{u}(y)\right|$ of the HVS (thick solid curve) contrasts well with that of NBW (thin solid curve), which is indicated to be kept at order 1 (as verified in Ref. [11]). 


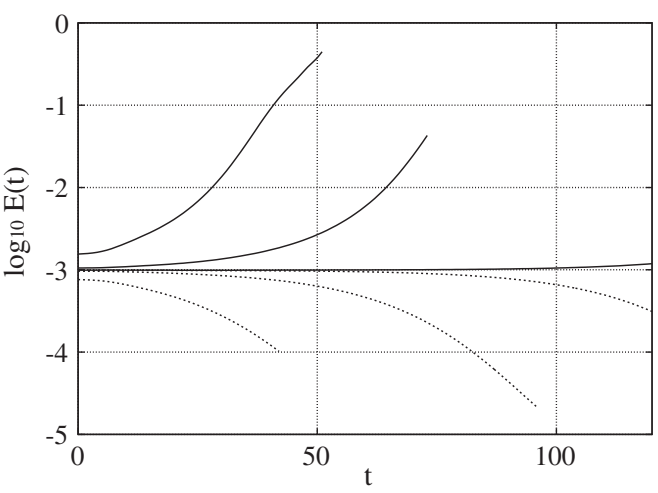

FIG. 2. $E(t)$ is half the norm of the wall-normal component of the perturbation at time $t$. Trajectories starting around $\boldsymbol{u}_{H}$ separate towards either the laminar (dotted) or the turbulent (solid) states due to a small additive perturbation onto the initial state. Time development is carried out under the periodic boundary conditions with $\left(L_{x}, L_{z}\right)=(2 \pi, \pi)$ by imposing the reflection symmetry on the perturbation.

for the turbulent regime. The closer the initial condition is to $\boldsymbol{u}_{H}$ (the smaller the perturbation is), the longer transitional interval it takes for $\boldsymbol{u}(t)$ to reach either turbulent or laminar regimes. This means that HVS remains on the BB, and that it is an unstable solution even under the restricted dynamical system.

Following these facts mentioned above, (1) the lower branch of HVS gets closer to the laminar with increasing Re and (2) the lower branch of HVS stays on BB even at high $\mathrm{Re}$, we can deduce the assumption that the minimum distance between the laminar state and BB might be representatively measured by $\left\|\boldsymbol{u}_{H}-\boldsymbol{u}_{L}\right\|$, which asymptotically vanishes with the increase of Re. Therefore, turbulent transition triggered by a small perturbation originating at HVS does not require an additional streamwise vortex component, even if the perturbation on the laminar state is infinitely small at the infinite Re. Note that this is in contrast to a conjecture on transition deduced from the asymptotic behavior of NBW concluded in Ref. [11]. In their conjecture, it is supposed that the formation of an $\mathrm{O}\left(\mathrm{Re}^{-1}\right)$ updraft necessarily creates $\mathrm{O}(1)$ streaks which in turn leads to turbulent transition via a certain nonlinear interaction between these modes.

The manifold of HVS expanding towards NBW.Additionally, we investigated one of manifolds of HVS for $\operatorname{Re}=1000$, again employing the edge tracking. Let us take a plane in state space on which the three distinct exact steady solutions of PCF, the laminar state, the lower branch of HVS, and the lower branch of NBW, lie. Using a couple of parameters $(a, b)$, we specify an arbitrary state $\boldsymbol{u}_{(a, b)}$ on the plane, which is expressed as the superposition of these solutions as follows:

$$
\boldsymbol{u}_{(a, b)}=b \boldsymbol{u}_{\mathrm{L}}+(1-b)\left((1-a) \boldsymbol{u}_{\mathrm{H}}+a \boldsymbol{u}_{\mathrm{N}}\right) .
$$

It should be noted that $\boldsymbol{u}_{(a, b)}$ satisfies the incompressible condition, but is not necessarily equivalent to a steady state of the governing equation. By adopting the state $\boldsymbol{u}_{(a, b)}$ for a different set of $(a, b)$ as an initial state, $\boldsymbol{u}(t=0)$, a number of trial calculations of the time development of the state $\boldsymbol{u}(t)$ are carried out, which will provide part of the outline of the BB.

Here, let us plot $\boldsymbol{u}(t)$ as a trajectory expressed by $(\xi(t), \eta(t), \zeta(t))$ in a three-dimensional space. The distance between two different flow states $\boldsymbol{u}_{1}$ and $\boldsymbol{u}_{2}$ in the state space could be measured by the norm of the difference of the associated velocity fields, $\left\|\boldsymbol{u}_{1}-\boldsymbol{u}_{2}\right\|$. If one plots the laminar state at the origin, and the HVS on the $\xi$ axis $(\xi>0)$ with the distance, $\left\|\boldsymbol{u}_{H}-\boldsymbol{u}_{L}\right\|$, from the origin of the space, then NBW may be plotted in the first quadrant on the $\xi-\eta$ plane so as to match the corresponding distances, $\left\|\boldsymbol{u}_{N}-\boldsymbol{u}_{L}\right\|$ and $\left\|\boldsymbol{u}_{N}-\boldsymbol{u}_{H}\right\|$, from the origin and HVS. Thus, an arbitrary state $\boldsymbol{u}$ can be plotted at a point in the space with the distances from the three exact

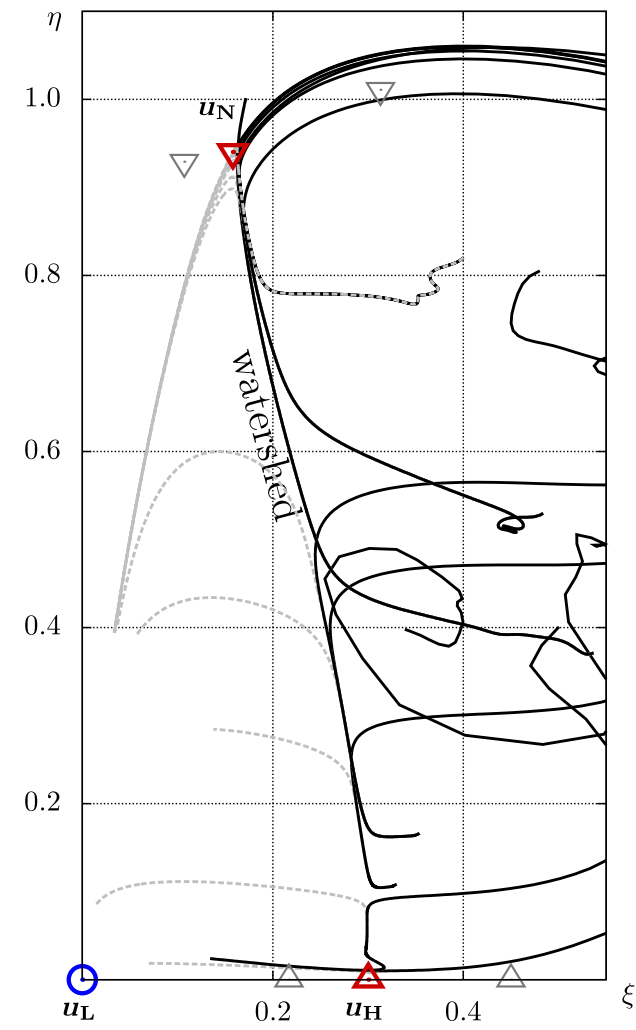

FIG. 3 (color online). Trajectories starting from initial states, $\boldsymbol{u}_{(a, b)}$ for a different set of parameters $(a, b)$, separate towards either the laminar $\boldsymbol{u}_{L}$ (grey dashed curves) or the turbulent states (black solid curves), which are obtained at $\mathrm{Re}=1000$. In the case of $\eta>0$, the initial state does not satisfy the reflection symmetry. Some of trajectories starting around $\boldsymbol{u}_{H}$ at $\operatorname{Re}=1000$ (red upward triangle) with a particular set of $(a, b)$ trace a part of the BB as "watershed," which divides the whole space into the basins of the turbulent and laminar attractors. This BB is outlined by a heteroclinic orbit [23] connecting $\boldsymbol{u}_{H}$ and $\boldsymbol{u}_{N}$ at $\operatorname{Re}=1000$ (red downward triangle). For reference, $\boldsymbol{u}_{H}$ and $\boldsymbol{u}_{N}$ obtained at $\operatorname{Re}=300,10000$ are plotted as well (grey triangles), both of which approach towards $\xi=0$ with increasing Re. 
states. Figure 3 is the projection of the trajectories, $\boldsymbol{u}(t)$, onto the $\xi-\eta$ plane in space. Generally speaking, since the real state space consists of much higher dimensions, any state cannot maintain its identity in the projection.

Trajectories for a variety of set values, $(a, b)$, obtained at $\operatorname{Re}=1000$, are plotted in Fig. 3. Note that calculations with a variety of $b$ under fixed $a=0$ were demonstrated above (see Fig. 2), which, if plotted, would be on the $\xi$ axis in Fig. 3. In the case of $a=0$, the state $\boldsymbol{u}(t)$ at a sufficient large $t$ decreases to $\boldsymbol{u}_{L}$ for $b<0$, whereas it develops to some turbulent state for $b>0$. In the figure, all trajectories starting from $\boldsymbol{u}_{H}$ head eventually towards either $\boldsymbol{u}_{L}$ or a turbulent state $\left(\xi^{2}+\eta^{2}>1\right)$, but some of them pass by $\boldsymbol{u}_{N}$. A watershed, on which both $\boldsymbol{u}_{H}$ and $\boldsymbol{u}_{N}$ lie, emerges out of a tangle of trajectories, as if a ridge would be outlined by a lot of small streams in a mountainous area on a map. The obtained watershed corresponds to the outline of the BB, which divides the whole space into the basins of the turbulent and laminar attractors, with the heteroclinic orbit [23] of these solutions constituting the BB.

Concluding remarks.-We will list briefly the facts obtained from the study on BB of PCF. First, the lower branch of HVS asymptotically approaches the laminar state with increasing Re. Second, the lower branch of HVS stays on the BB at high Re. Third, while one of the unstable manifolds of HVS connects to the laminar state, another unstable manifold of HVS connects to the lower branch NBW, that is, a heteroclinic orbit of these solutions constitutes the BB. Additionally, NBW is a robust attractor in $\mathrm{BB}$ even at high $\mathrm{Re}$, which is a conclusion given in Ref. [11].

From all four above facts, we may deduce an ideal process of turbulent transition from the dynamical point of view. In experiments of the turbulent transition, the magnitude of perturbation on the laminar is reduced as much as possible at the initial stage in the upstream. In case the flow experiences turbulent transition, though, the adopted smallest perturbation necessarily satisfies the reflection symmetry, which is inferred from the first and the second points. From the third point, we may expect that the trajectory tends to approach towards NBW along the heteroclinic orbit on BB. In the downstream, NBW-type structures would be prevalent, while HVS is observed rarely, which is suggested from the fourth point. This scenario gives us a clue to answer the question, why the vortex structure with the spanwise reflection symmetry is often observed at a transition stage in the turbulent boundary layer with a sufficiently large Re, where what is observed no longer depends on Re. At the same time, this does not contradict that a meandering streaky structure like NBW rather than HVS would be ubiquitous downstream in fully developed turbulent shear flows (cf. [18]). Moreover, the lower branch of HVS, rather than that of NBW, may give us a more practical criteria of the laminarturbulent transition of PCF at high Re. An interpretation of these results, that a state satisfying the reflection symmetry (varicose mode) is more optimal than its counterpart (sinuous mode) to trigger transition, is reasonably supported by the exhaustive measurements of the minimal distance from the laminar state to BB of Ref. [24].

T. A. was supported by the European Union FP7 people Marie Curie International Incoming Fellowship Grant No. T2T-VDG F7-PEOPLE-2011-IIF 298891. This work has also been supported in part by KAKENHI (No. 23760164) and by the Kansai University Special Research Fund 2012.

*Corresponding author. itano@kansai-u.ac.jp

${ }^{\dagger}$ Corresponding author. s.c.generalis@aston.ac.uk

[1] J. F. Gibson, J. Halcrow, and P. Cvitanovic, J. Fluid Mech. 638, 243 (2009).

[2] T. Itano and S. C. Generalis, Phys. Rev. Lett. 102, 114501 (2009).

[3] G. Kawahara, M. Uhlmann, and L. van Veen, Annu. Rev. Fluid Mech. 44, 203 (2012).

[4] H. Wedin and R. R. Kerswell, J. Fluid Mech. 508, 333 (2004).

[5] M. Nagata, J. Fluid Mech. 217, 519 (1990).

[6] R. M. Clever and F. H. Busse, J. Fluid Mech. 344, 137 (1997).

[7] F. Waleffe, Phys. Rev. Lett. 81, 4140 (1998).

[8] V. Romanov, Funct. Anal. Appl. 7, 137 (1973).

[9] O. Dauchot and F. Daviaud, Phys. Fluids 7, 335 (1995).

[10] A. Monokrousos, A. Bottaro, L. Brandt, A. Di Vita, and D. S. Henningson, Phys. Rev. Lett. 106, 134502 (2011).

[11] J. Wang, J. Gibson, and F. Waleffe, Phys. Rev. Lett. 98, 204501 (2007).

[12] J. Del Álamo and J. Jiménez, Annual Research Briefs 2001 (Center for Turbulence Research) (Stanford University, Stanford, 2001), p. 329.

[13] Y. Hwang and C. Cossu, Phys. Rev. Lett. 105, 044505 (2010).

[14] T. Herbert, Annu. Rev. Fluid Mech. 20, 487 (1988).

[15] S. K. Robinson, Annu. Rev. Fluid Mech. 23, 601 (1991).

[16] R. J. Adrian, Phys. Fluids 19, 041301 (2007).

[17] X. Wu and P. Moin, J. Fluid Mech. 630, 5 (2009).

[18] P. Schlatter, R. Örlü, Q. Li, G. Brethouwer, A. V. Johansson, P.H. Alfredsson, and D.S. Henningson, in Progress in Simulations of Turbulent Boundary Layers, Sweden (University of Ottawa, Ottawa, Canada, 2011).

[19] S. C. Generalis and T. Itano, Phys. Rev. E 82, 066308 (2010).

[20] K. Deguchi and M. Nagata, Phys. Rev. E 82, 056325 (2010).

[21] B. Eckhardt, T. M. Schneider, B. Hof, and J. Westerweel, Annu. Rev. Fluid Mech. 39, 447 (2007).

[22] T. Itano and S. Toh, J. Phys. Soc. Jpn. 70, 703 (2001).

[23] J. Halcrow, J. F. Gibson, P. Cvitanovic, and D. Viswanath, J. Fluid Mech. 621, 365 (2009).

[24] Y. Duguet, L. Brandt, and B. R. J. Larsson, Phys. Rev. E 82, 026316 (2010). 